\title{
Kanizsa-type subjective contours do not guide attentional deployment in visual search but line termination contours do
}

\author{
Xingshan Li and Kyle R. Cave \\ University of Massachusetts, Amherst, Massachusetts \\ AND \\ JEREMY M. WOLFE \\ Brigham and Women's Hospital, Boston, Massachusetts \\ and Harvard Medical School, Boston, Massachusetts
}

\begin{abstract}
We used visual search to explore whether attention could be guided by Kanizsa-type subjective contours and by subjective contours induced by line ends. Unlike in previous experiments, we compared search performance with subjective contours against performance with real, luminance contours, and we had observers search for orientations or shapes produced by subjective contours, rather than searching for the presence of the contours themselves. Visual search for one orientation or shape among distractors of another orientation or shape was efficient when the items were defined by luminance contours. Search was much less efficient among items defined by Kanizsa-type subjective contours. Search remained efficient when the items were defined by subjective contours induced by line ends. The difference between Kanizsa-type subjective contour and subjective contours induced by line ends is consistent with physiological evidence suggesting that the brain mechanisms underlying the perception of these two kinds of subjective contours may be different.
\end{abstract}

In this article, we revisit the interaction of visual attention and subjective contours (Davis \& Driver, 1994, 1998; Grabowecky \& Treisman, 1989; Gurnsey, Humphrey \& Kapitan, 1992). Specifically, we ask whether subjective contours can guide the deployment of attention in visual search tasks. Our answer will be that it depends on the nature of the subjective contour. Items defined by subjective contours produced by line endings can effectively guide attention. Items defined by Kanizsa-type ( pacman) subjective contours fail to guide attention.

We are far from the first to address this question. In a number of studies, the relationship between subjective contours and attention has been investigated using visual search tasks. In typical visual search tasks, observers search for a target in displays containing varying numbers of distractor items. The efficiency of the search can be assessed by varying the number of items (the set size) and measuring reaction time (RT). In highly efficient searches (e.g., for a red item among green distractors), attention can be deployed to the target with little regard for the number of distractor items (e.g., Green \& Anderson, 1956). The slope of the resulting RT $\times$ set size function will be near zero. Other searches (e.g., a search for a "T" among "L"s) are inefficient, with RT $\times$ set size slopes of 20-30 msec/item for target-present trials and something more than twice that for trials on which the target is absent
(Wolfe, 1998). This is true even if the items can be identified without the need for eye movements. If each item must be fixated, search is far less efficient.

Between the extremes of efficient and inefficient search lies a continuum of tasks for which search efficiency is determined by how effectively basic features can be used to guide attention to the target (Cave, 1999; Wolfe, Horowitz, \& Michod, 2007). Thus, if observers search for a red "T" among "L"s that can be either red or black, the color information will guide attention to the red items, reducing the effective set size and reducing the slope of the RT $\times$ set size function (Egeth, Virzi, \& Garbart, 1984).

A number of approaches have been used to determine whether subjective contours can guide attentional deployment. Grabowecky and Treisman (1989) had participants search for a subjective triangle among distactors, which were sets of three pacmen that were misaligned so that they did not induce a subjective figure. The logic of the experiment was that the presence of a subjective contour might pop out in the way that a red item pops out among green items. However, when the results showed that RT increased with set size, they concluded that visual search for subjective contours was serial (or, in our more theoretically neutral language, inefficient) and, so, subjective contour perception was a process requiring attention. This finding can be seen as part of a long-standing debate over 
whether subjective contours are produced bottom up, in a stimulus-driven fashion (Shapley \& Gordon, 1985), or top down, with input from higher level cognitive representations (Rock \& Anson, 1979; von der Heydt, 1987; see Lesher, 1995, for a review).

The conclusion that subjective contours required attention was questioned by Gurnsey et al. (1992; see below) and Davis and Driver (1994). Davis and Driver (1994) argued that Grabowecky and Treisman's (1989) slow detection of subjective contour may have been caused by the sudden onset of the pacmen. They designed an experiment to control the sudden onset effect. Nine hundred milliseconds before the onset of each search display, placeholders were presented at the locations that would be occupied by the pacmen that would induce the subjective contours. All the clusters, which were separated from each other, were located along an imaginary circle whose center was the location of fixation. The task was to search for a subjective square. Davis and Driver (1994) found quite efficient slopes as a function of set size (about $10 \mathrm{msec} / \mathrm{cluster}$ ). From these results, they concluded that subjective contour perception was preattentive. In other words, Kanizsa-type subjective contours can guide the deployment of visual attention.

In turn, Gurnsey, Poirier, and Gascon (1996) argued that there were many factors that distinguished the targets and the distractors in Davis and Driver's (1994) experiments besides the presence of subjective contours. For example, the targets and the distractors differed in gross outline and closure. They designed experiments that showed that when subjective contours were eliminated, visual search was still fast and efficient; and when subjective contours were presented in some conditions, RT showed a steep increase with set size. They concluded that subjective contours played no role in the parallel search in Davis and Driver's (1994) results. Although Gurnsey et al.'s (1996) study raised doubts about Davis and Driver's (1994) conclusions, it did not provide a final answer as to whether Kanizsa-type subjective contour can guide attention.

Davis and Driver (1998) also noted the potential shortcomings in their own 1994 experiment. They suspected that parallel search in Davis and Driver (1994) may have resulted from strong grouping between the four pacmen in a target cluster. To overcome these shortcomings, Davis and Driver (1998) designed a series of experiments. Their displays consisted of sets of clusters of four black pacmen each, with the pacmen arranged so as to generate a Kanizsa-type subjective square between them. In some of the clusters, one of the black pacmen at one corner of the square was replaced by either a large brown circle or a notched brown circle with a $90^{\circ}$ segment taken out of it. Stereoscopic displays were used so that different components of the stimulus array would appear in different depth planes. The task was to search for a large brown notched circle among large brown complete circles. Their results showed that visual search was inefficient if the notched object and distractors were behind an abutting subjective-square surface but was efficient if the notched object and distractors were in front of the subjectivesquare surface. They explained the results by assuming that when the notched target appeared to lie behind an abutting subjective-square surface, the incomplete target circle became amodally completed and, thus, was difficult to distinguish from the complete brown nontarget circles preattentively. Since the amodally completed circle behaved like a complete circle, they concluded that Kanizsatype subjective contour was generated prior to the search for the notched circle and, presumably, without the need for attention to the inducers.

Davis and Driver's (1998) evidence in favor of the preattentive status of subjective contours is a little indirect-at least, if one's primary interest is in the ability of subjective contours to guide attention in visual search. Since Gurnsey et al.'s (1996) critique of Davis and Driver's (1994) visual search results, there have been several other claims for efficient search for pacman-style Kanizsa figure among clumps of pacmen that did not form subjective squares or triangles (Herrmann \& Mecklinger, 2000; Senkowski, Röttger, Grimm, Foxe, \& Herrmann, 2005; Takahashi, Ohya, Arakawa, \& Tanabe, 2007). In all these cases, observers detected the presence of a subjective figure among collections of inducers that did not produce the figure. This always leaves open the possibility that something other than the contour itself differentiated target groups of pacmen from distractor groups (Gurnsey, personal communication, August 6, 2007). Accordingly, in our experiments, we asked whether a bar of one orientation pops out of bars of another orientation when those bars are produced by subjective contours. In this case, all the pacmen in all groups face "inward," eliminating that class of possible confounds.

Gurnsey et al. (1992) explored the relation between visual attention and a second type of subjective contoursthose induced by offset gratings or line endings. They found that search was efficient when the target was a vertical subjective bar among horizontal subjective bars and was efficient when the target was a subjective crescent among subjective oblique bars. They concluded that subjective contours defined by offset gratings could be detected in parallel. In the present work, we directly compared these two types of subjective contours, equated for their perceptual salience; as is indicated in the title, we will report that they produced different patterns of results.

\section{EXPERIMENTS WITH KANIZSA-TYPE SUBJECTIVE CONTOURS}

\section{Experiment 1}

Most of the visual search experiments involving Kanizsa-type contours have emphasized the detection of the subjective contour and/or figure: Can observers find a subjective target among distractors that do not generate subjective contours? In this experiment, we asked a somewhat different question: Can secondary properties of subjective figures efficiently guide attention? Our approach is analogous to that of Cavanagh, Arguin, and Treisman (1990). They asked whether observers could search for a vertical bar among horizontal bars if that bar was generated by something other than a luminance contour. They found that observers could search efficiently for an oblique bar 
among vertical bars when the bars were defined by color, texture, relative motion, and, more equivocally, binocular disparity. Here, we asked whether the bar could be defined by Kanizsa contours. In two control conditions, real lines appeared at the locations that would otherwise have manifested subjective contours.

\section{Method}

Apparatus. The stimuli were presented on a 19-in. NEC MultiSync FE990 monitor controlled by a Mac G4. The participants kept their chin on a chinrest located $57 \mathrm{~cm}$ away from the monitor. The participants responded by pressing a button on a Superlab button box.

Participants. All of the participants were recruited from a participant pool of undergraduate students at the University of Massachusetts, who participated for course credit. All the participants had normal or corrected-to-normal vision. No participant participated in more than one of the following experiments. The average age was 20.3 years, ranging from 18 to 28 . There were 18 participants in Experiment 1 .

Stimuli. The task in this experiment was to search for a horizontal rectangle target among vertical rectangle distractors. There were three blocks of trials in Experiment 1. In one block, the rectangles were defined by real contours alone. In another block, they were defined by subjective contours created by pacmen. Another block included both real lines and pacmen together, so that the rectangles were defined by both real and subjective contours (Figure 1). These conditions will be labeled as line, subjective, and both conditions, respectively. The order of these blocks was balanced so that the same number of participants was tested in each possible order.

The diameter of each pacman was $16.8 \mathrm{~mm}$, or $1.69^{\circ}$ of visual angle. The size of the induced rectangle was $25.2 \times 37.8 \mathrm{~mm}$, or $2.53^{\circ} \times 3.79^{\circ}$ of visual angle. To prevent the density of the pacmen from differing too much for different set sizes, the following method was employed when producing stimuli. The location of the first rectangle was selected randomly. The location of the next rectangle was chosen so that one of its inducing pacmen was no farther than $25.2 \mathrm{~mm}$ from one of the existing pacmen and no nearer than $20.0 \mathrm{~mm}$ from any of the existing pacmen. Each additional pacman was positioned in the same way. This design ensured that the pacmen appeared in a "clump" with a density that did not vary across the different set sizes. Because the first pacman was selected randomly, the stimuli could appear anywhere on the display. The eccentricity of the clump was random, so that eccentricity did not vary systematically across set sizes. The black pacmen $\left(0 \mathrm{~cd} / \mathrm{m}^{2}\right)$ were displayed on a white background $\left(86 \mathrm{~cd} / \mathrm{m}^{2}\right)$. In the both and line conditions, the real contours were gray $\left(16 \mathrm{~cd} / \mathrm{m}^{2}\right)$. As is shown in Figure 1, the real contours were deliberately quite weak, in an effort to mimic the low subjective contrast of subjective contours.

There were four different set sizes in the search array: 5, 7, 9, and 13. In half of the trials, a horizontal rectangle was presented as the target. Thus, there were two (target present or not) $\times$ four (set size) conditions in each block. There were 10 trials for each condition, or 80 trials in each block of the experiment, making a total of 240 trials for the entire experiment. The order of the 80 trials was randomized within each block. Before each block, there were 32 practice trials.

Procedure. At the beginning of each trial, a red cross appeared at the center of the screen for $1 \mathrm{sec}$. Gurnsey et al. (1992) and Davis and Driver (1994) argued that the sudden onset of high-contrast-inducing pacmen may have overpowered any signal for the subjective target in Grabowecky and Treisman's (1989) study. Following Davis and Driver (1994), placeholders were presented for $1 \mathrm{sec}$ at the positions that would later be occupied by pacmen. Davis and Driver (1994) used black circles as placeholders, with one quarter of each circle removed when the time came to induce the subjective contours. Because this sudden offset of the quarter of the black circle may have attracted attention to that part of stimuli, we used placeholders with the same shape and same location as the pacmen, but with a random rotation applied to each. After an exposure of $1 \mathrm{sec}$, the inducers were all simultaneously rotated to the correct orientation to induce the subjective contours. In the line condition, the placeholders disappeared after $1 \mathrm{sec}$, and the rectangle stimuli appeared drawn with real lines,
A

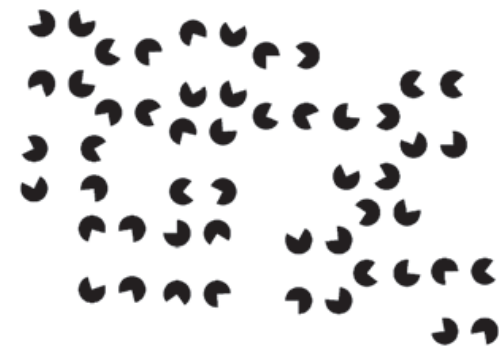

C

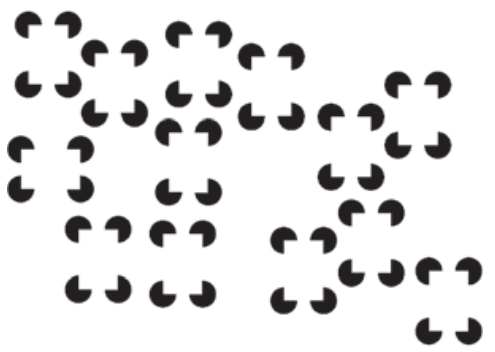

B

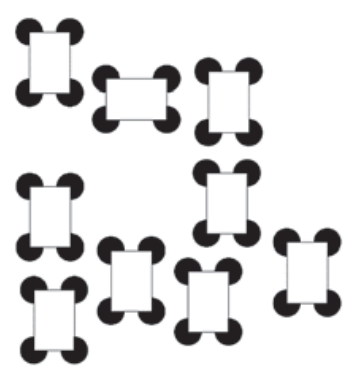

D

Figure 1. Stimuli in Experiment 1. (A) Placeholders that appeared at the beginning of a trial in all three conditions. (B) Stimulus from the both condition. (C) Stimulus from the subjective condition. (D) Stimulus from the line condition. 
with no inducers present. In all three conditions, the search array was presented until the participants responded.

The participants responded by pressing the right button on the button box if the target was present and the left button if the target was absent. The participants were instructed to respond as quickly and as accurately as possible. An error response was indicated by a sound.

\section{Results}

The overall accuracy was $97.2 \%$. The accuracy was $97.8 \%, 97.5 \%$, and $91.5 \%$ for the both, line, and subjective conditions, respectively, when the target was present and was $98.8 \%, 99.1 \%$, and $99.4 \%$ when the target was absent. Because the accuracy was very high, it was not analyzed further. Only trials with correct responses were included in the analyses, which excluded 120 trials $(2.8 \%)$ among 4,320 trials. Trials with RTs shorter than $100 \mathrm{msec}$ or longer than $10,000 \mathrm{msec}$ were also excluded, which resulted in an additional 7 trials being excluded.

The key results are shown in Figure 2. For the both condition, the slope of the function relating mean RT and set size was $10.6 \mathrm{msec} / \mathrm{rectangle}$ when the target was present and $58.2 \mathrm{msec} /$ rectangle when the target was absent. In contrast, in the subjective condition, the target-present slope was $52.2 \mathrm{msec} / \mathrm{rectangle}$, and the target-absent slope was $115.1 \mathrm{msec} /$ rectangle. The ratio for target absent versus target present was about 2.2. In the line condition, the target-present slope was $18.2 \mathrm{msec} /$ rectangle, and the target-absent slope was $43.5 \mathrm{msec} /$ rectangle.

A series of pairwise $t$ tests was conducted on the search slope data, derived by linear regressions of the mean RTs against set size for each participant. For target-present trials, the slopes were significantly different between the both and the subjective conditions $[t(17)=-4.47, p<.01$, Cohen's $d=-1.45]$; and between the line and subjective conditions $[t(17)=-4.10, p<.01, d=-1.10]$. However, the slopes for the both and the line conditions did not differ significantly $[t(17)=-1.72, p>.1, d=-0.48]$. For the target-absent condition, all of the pairs of slopes were significantly different $[t \mathrm{~s}=2.75,-5.90$, and -6.49 , and $d=0.40,-1.05$, and -1.42 , for the both and line, the both and subjective, and the line and subjective conditions, respectively; $p \mathrm{~s}<.05]$.

\section{Discussion}

Can visual attention be guided to a bar of unique orientation if that orientation is defined by a subjective contour of the Kanizsa variety? The results of Experiment 1 sug- gest not. It is clear that the search in the subjective condition was very inefficient. It was more inefficient than the search for a oriented bar defined by a weak luminance edge (the line condition). The subjective contour search met the usual standard for serial search: target-present slopes of 20-30 msec or more and a ratio of target-absent to target-present slopes that was somewhat greater than 2:1 (Wolfe, 1998). It is notoriously difficult to use data of this sort to determine whether the underlying process was parallel or serial (Townsend, 1990) or, for that matter, a hybrid (Wolfe, 2003). Regardless, it is apparent that search in the both condition was much more efficient than that in the subjective condition, reflecting a strong difference between the ability of subjective contours and real contours to guide the deployment of attention.

One may argue that the inefficient search in the subjective condition was due to retinal eccentricity. The decrease in spatial resolution in the periphery may have prevented the participants from perceiving the edges of the inducing pacmen when they were at the edges of the display. Search may have been more difficult for the larger set sizes in these experiments merely because there were more stimuli at extreme eccentricities (Carrasco \& Frieder, 1997). To preclude this possibility, the target-present trials were subjected to two other analyses. In the first analysis, these trials were divided into three equal-size categories based on the eccentricity of the target. The ranges of the categories were $0^{\circ}-5.3^{\circ}, 5.3^{\circ}-8.9^{\circ}$, and $8.9^{\circ}-15.9^{\circ}$. The slope data for the search were submitted to an ANOVA, with the different stimuli types (both, subjective, or line) and categories of eccentricity as within-subjects factors. The effect of eccentricity was not significant $\left[F(2,36)=1.95, p>.1, \eta_{\mathrm{p}}^{2}=\right.$ $0.10]$. Moreover, there was no interaction between eccentricity and stimulus type $(F<1)$. In the second analysis, RT slope as a function of eccentricity was calculated for each participant in each stimulus condition. An ANOVA showed no main effect of stimulus type $[F(2,34)=1.88, p>.1]$. These two analyses showed that eccentricity did not influence search performance differently for different stimulus types. Therefore, the eccentricity of the stimuli was not a key factor in the pattern of results in Experiment 1.

\section{Experiment 2}

In Experiment 1, both targets and distractors were rectangles. In Experiment 2, we used squares and triangles to
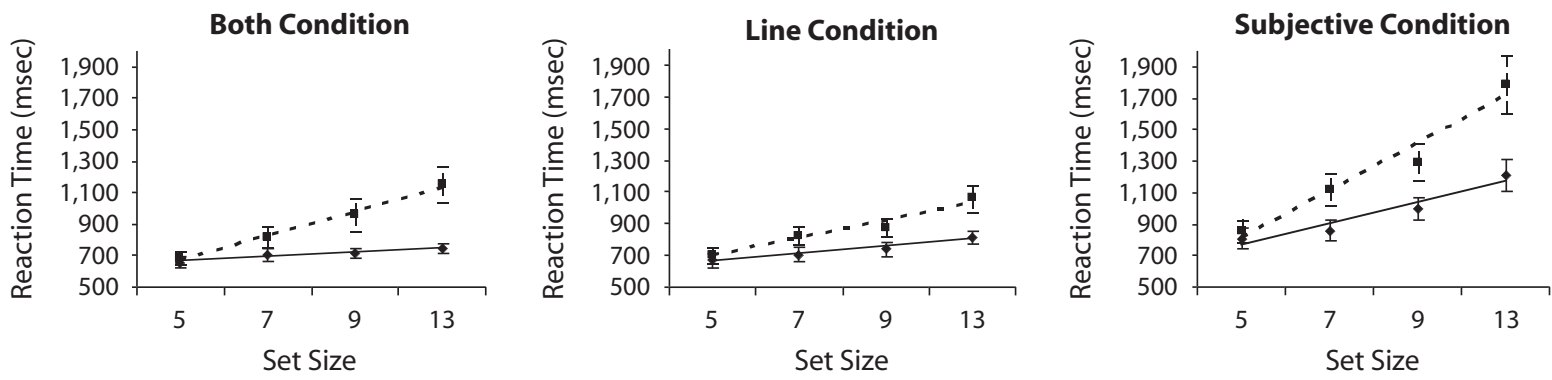

Figure 2. Reaction time $\times$ set size functions for each condition in Experiment 1. Target-present trials are indicated by solid lines, and target-absent trials by dashed lines. 
explore whether we could find a similar pattern of search performance when the target was defined by shape, rather than by orientation.

\section{Method \\ Stimuli and Procedure. In this experiment, the task was to search for a square among triangles. The stimuli are shown in Fig- ure 3 . To exclude the possibility that the shape of the pacmen plays some important role in visual search, the shape was modified so that all the pacmen, whether they induced a rectangle or a triangle, were identical in shape. Each pacman had two gaps: One was $90^{\circ}$, to cre- ate the vertex of a rectangle; the other was $60^{\circ}$, to create the vertex of an equilateral triangle. All of the other aspects of the experiment were identical to those in Experiment 1. Another 18 participants were recruited from the same participant pool.}

\section{Results}

One participant was excluded from the analysis because of extremely long RTs. The mean RT for all the participants was $711 \mathrm{msec}$, and the standard deviation was $230 \mathrm{msec}$. The excluded participant had a mean RT of $1,430 \mathrm{msec}$, and the next largest mean RT was 974 msec.

The overall accuracy in this experiment was $97.2 \%$. Accuracy was $96.8 \%, 95.7 \%$, and $95.7 \%$ for the both, line, and subjective conditions, respectively, when the target was present and was $99.1 \%, 98.2 \%$, and $98.2 \%$ when the target was absent.

The results are shown in Figure 4. For the both condition, the slope of the RT function was $6.11 \mathrm{msec} / \mathrm{shape}$ when the target was present and $32.59 \mathrm{msec} / \mathrm{shape}$ when the target was absent. In contrast, for the subjective condition, the target-present slope was $20.80 \mathrm{msec} / \mathrm{shape}$, and the target-absent slope was $53.52 \mathrm{msec} / \mathrm{shape}$. For the line condition, the target-present slope was $8.51 \mathrm{msec} / \mathrm{shape}$, and the target-absent slope was $25.03 \mathrm{msec} / \mathrm{shape}$.

A series of pairwise $t$ tests were conducted on the search slope data. For target-present trials, the slopes were significantly different between the both and the subjective conditions $[t(16)=-3.83, p<.01, d=-1.28]$ and between the line and the subjective conditions $[t(16)=$ $-3.98, p<.01, d=-1.32]$. However, the slopes were not significantly different between the both and the line conditions $[t(16)=-0.79, p>.1]$. When the target was absent, the pairs of slopes were significantly different between the both condition and the subjective condition $[t(16)=-2.73, p<.05, d=-0.64]$ and between the line condition and the subjective condition $[t(16)=-3.54$, $p<.01, d=-1.03]$. However, the slopes were not significantly different between the both and the line conditions $[t(16)=1.37, p>.1]$.

\section{Discussion}

Although different stimuli were used, the result of Experiment 2 had a pattern similar to that in Experiment 1. Search was difficult and strongly affected by set size in the subjective condition but was much more efficient in the both condition. This result confirmed the conclusion in Experiment 1 that Kanizsa-type subjective contours cannot guide the deployment of attention.

In this experiment, all of the slopes were smaller than the corresponding slopes in Experiment 1, reflecting the fact that the search task in Experiment 2 was generally easier than that in Experiment 1.

\section{Experiment 3}

In the first two experiments, placeholders were presented before the search array. It may be argued that this procedure can cause the perception of motion and, hence, interfere with the following search performance. Experiment 3 was designed to study the role of the placeholders in the first two experiments.

\section{Method}

Stimuli and Procedure. Seventeen participants were tested in this experiment. The background was gray $\left(57 \mathrm{~cd} / \mathrm{m}^{2}\right)$ to allow comparison between this experiment and a later experiment. This change should not change the relationship between the both and the subjective conditions. All other aspects of this experiment were similar to those in Experiment 1, except for the absence of placeholders.

\section{Results}

The results are shown in Figure 5. For the both condition, the slope of the function relating mean RT and set size was $6.77 \mathrm{msec} /$ rectangle when the target was present and $60.97 \mathrm{msec} / \mathrm{rectangle}$ when the target was absent. In contrast, for the subjective condition, the target-present slope was $37.12 \mathrm{msec} /$ rectangle, and the target-absent slope was $91.21 \mathrm{msec} / \mathrm{rectangle}$. The ratio for target absent
A

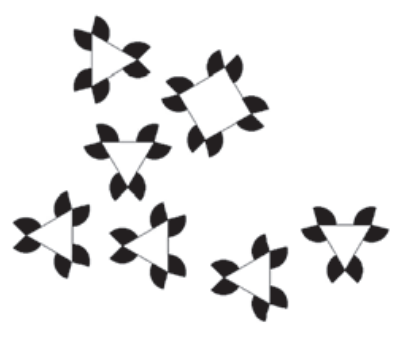

B

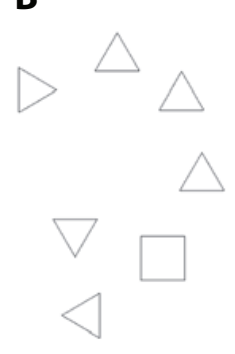

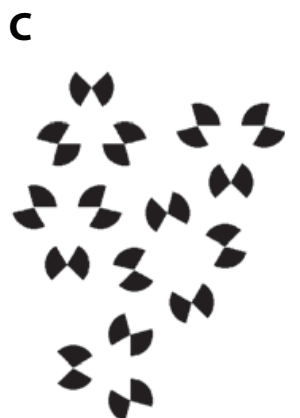

Figure 3. Stimuli in Experiment 2. (A) Both condition. (B) Line condition. (C) Subjective condition. 

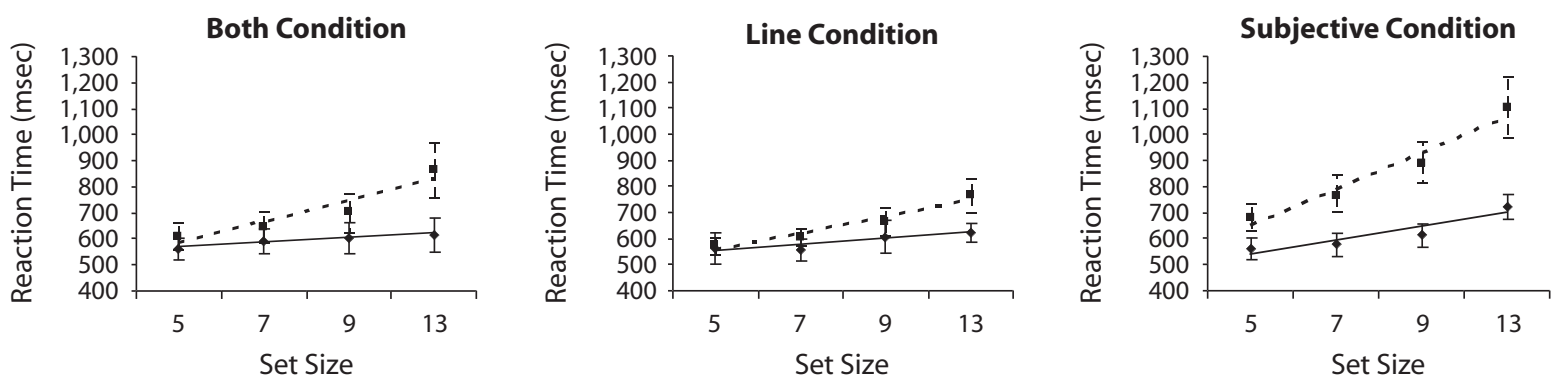

Figure 4. Reaction times in Experiment 2. Target-present trials are indicated by solid lines, and target-absent trials by dashed lines.

versus target present was about 2.5 . For the line condition, the target-present slope was $8.96 \mathrm{msec} /$ rectangle, and the target-absent slope was $41.27 \mathrm{msec} /$ rectangle.

A series of pairwise $t$ tests was conducted on the search slope data. For target-present situations, the slopes were significantly different between the both and the subjective conditions $[t(16)=-5.69, p<.01, d=-1.71]$ and between the line and subjective conditions $[t(16)=-4.35$, $p<.01, d=-1.49$ ]. However, the slopes were not significantly different between the both and the line conditions $[t(16)=-0.73, p>.1]$. For the target-absent condition, all of the pairs of slopes were significantly different $[\mathrm{ts}(16)=$ $2.13,-3.54$, and -4.62 , and $d \mathrm{~s}=0.60,-0.70$, and -1.27 for the both and line, the both and subjective, and the line and subjective conditions, respectively; $p$ s $<.05$ ].

\section{Discussion}

Even without placeholders, the results of this experiment had the same pattern as that in Experiment 1. The visual search was quite efficient in the both and line conditions but was much more inefficient in the subjective condition. This makes it unlikely that the patterns of results in Experiments 1 and 2 were due to the use of placeholders.

\section{Experiment 4}

One of the differences between Experiments 1 and 2 and Davis and Driver's (1994) experiment was the visual angle of the search array presented on the screen. To exclude retinal eccentricity as an explanation for the results of the first two experiments, Experiment 4 displayed stimuli within a visual angle that was comparable to that in Davis and Driver (1994), who found efficient visual search with Kanizsa-type subjective contour. The search array was limited to a much smaller region surrounding the fovea; this change greatly reduced the eccentricity of the search array. If the lack of effective search in the first two experiments was due to the difficulty in finding targets far from the fovea, we would expect to find a more efficient search in the subjective condition in this experiment and less difference between the subjective condition and the both condition.

\section{Method}

Stimuli and Procedure. The viewing distance was $112 \mathrm{~cm}$. The stimuli were displayed within an $88.15 \times 88.15 \mathrm{~mm}$ square at the center of the monitor. This square extended over a visual angle of $4.5^{\circ} \times 4.5^{\circ}$. Each pacman was $0.4^{\circ}$ in diameter. Because of the limited display area, set sizes of 5, 7, and 9 were used. All of the other aspects of this experiment were exactly the same as those in Experiment 1 . Eighteen participants from the same participant pool, who had not taken part in the first three experiments, were recruited to participate in this experiment. The stimuli differed from those in Davis and Driver (1994) in (1) gross outline and closure, (2) grouping, and (3) set size. The pacmen faced inward to induce the target in Davis and Driver's (1994) stimuli, whereas the pacmen faced outward to produce the distractor. Hence, the target differed from the distractors by gross outline and closure in Davis and Driver's (1994) stimuli, but there were no such differences between the present target and the distractors. The items were grouped into four item clusters and were placed in an imaginary circle in Davis and Driver's (1994) experiment, but not here. The set size was 2, 4, and 6 in Davis and Driver (1994); here the set size was 5, 7, and 9.

\section{Results}

The results are shown in Figure 6. In the both condition, the slope of the mean RT as a function of set size was $8.23 \mathrm{msec} / \mathrm{rectangle}$ when the target was present and
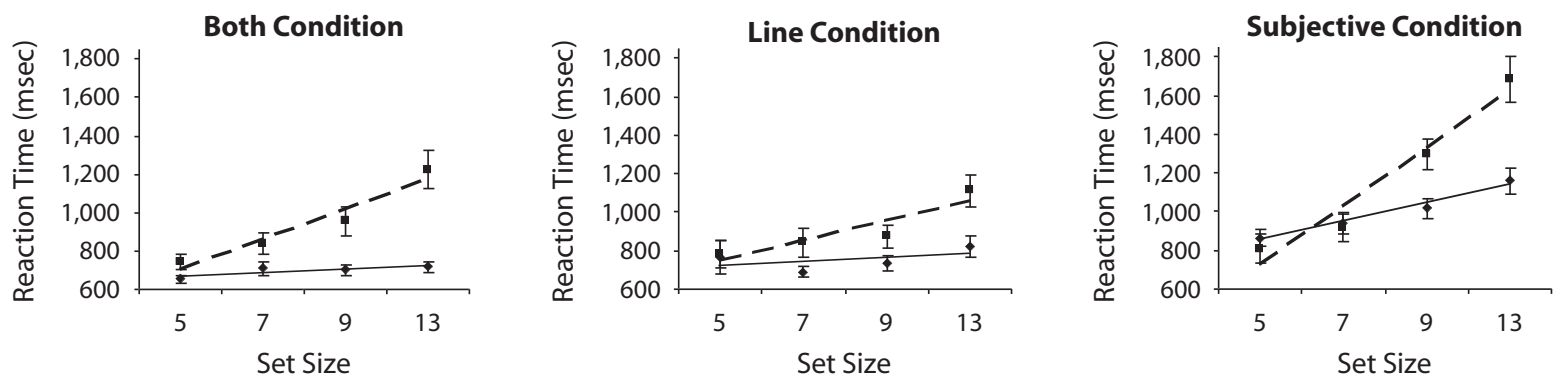

Figure 5. Reaction times in Experiment 3. Target-present trials are indicated by solid lines, and target-absent trials by dashed lines. 
$21.62 \mathrm{msec} /$ rectangle when the target was absent. In the line condition, the target-present slope was $23.09 \mathrm{msec} /$ rectangle, and the target-absent slope was $15.28 \mathrm{msec} /$ rectangle. In contrast, in the subjective condition, the target-present slope was $65.97 \mathrm{msec} / \mathrm{rectangle}$, and the target-absent slope was $94.94 \mathrm{msec} /$ rectangle.

A series of pairwise $t$ tests were conducted on the search slope data. For target-present situations, the slopes were significantly different between the both and the subjective conditions $[t(17)=-4.05, p<.01, d=-1.18]$ and between the line and the subjective conditions $[t(17)=-2.42, p<$ $.05, d=-0.85]$. The slopes were marginally significantly different between the both and the line conditions $[t(17)=$ $-1.97, p<.1, d=-0.62]$. For the target-absent condition, the slopes were significantly different between the both and the subjective conditions and between the line and the subjective conditions $[t \mathrm{~s}(17)=-8.26$ and -7.16 , respectively, $p \mathrm{~s}<.01, d \mathrm{~s}=-1.94,-2.07]$. The slopes were marginally different between the both condition and the line condition $[t(17)=2.02, p<.1, d=0.39]$.

\section{Discussion}

Although the stimuli were limited to a region that encompassed only $4.5^{\circ} \times 4.5^{\circ}$ visual angle, we found the same pattern of results as in Experiment 1. The search was much more efficient in the both and line conditions than in the subjective condition. As compared with the results of Experiment 1 , the slope was smaller in the both condition, and the slope was larger in the line and subjective conditions. This showed that the inefficient search in the subjective condition in Experiment 1 was not due to eccentricity.

\section{Experiment 5}

All of the experiments above utilized Kanizsa-type contours with high-contrast pacmen as inducing stimuli. Gurnsey et al. (1992) proposed that Kanizsa-type subjective contour perception might be impaired so much by surrounding high-contrast pacmen that attention was needed to enhance the perception of subjective contour. This experiment was designed to explore how the contrast of the contour in the both and subjective conditions would influence search performance.

\section{Method}

Stimuli and Procedure. Seventeen participants took part in this experiment. Each participant performed five blocks of trials, each with a different stimulus type. One block was the subjective condition. The other four were the both conditions, with the contours in each block having a different gray level: 6, 16, 39, and $60 \mathrm{~cd} / \mathrm{m}^{2}$. These gray levels produced a wide range of different contrasts-87\%, 69\%, 38\%, and $18 \%$ - which will be labeled both 87 , both 69 , both 38 , and both18. The gray level of the real contour in the both69 condition was similar to that of the real contour used in the first four experiments. The set sizes were 5, 7,9 , and 13. In half of the trials, the target was present. With the variations in set size, stimulus type, and target presence, there were 40 conditions in this experiment, with 8 trials for each condition. Thus, there were 320 experimental trials for each participant. At the beginning of each block, there were 32 practice trials. All of the other aspects of this experiment were exactly the same as those in Experiment 1.

\section{Results}

The RT slopes for the both87, both69, both38, and both 18 conditions were $14.26,9.96,12.89$, and $15.56 \mathrm{msec} /$ item, respectively, for the four gray levels when the target was present and 34.36, 26.40, 35.84, and $49.32 \mathrm{msec} / \mathrm{item}$ when the target was absent. In contrast, for the subjective condition, the target-present slope was $34.74 \mathrm{msec} /$ item, and the target-absent slope was $81.80 \mathrm{msec} /$ item.

Pairwise $t$ tests were conducted on the search slopes. For the target-present conditions, the subjective condition slope differed significantly from each of the both condition slopes $[t \mathrm{~s}(16)=-3.50,-3.67,-2.93$, and -3.52 , respectively; $d \mathrm{~s}=-0.92,-1.12,-0.95$, and -0.85 ; all $p \mathrm{~s}<.01]$. These differences were also significant when the target was absent $[t \mathrm{~s}(16)=-5.87,-6.27,-4.94$, and -3.85 , respectively; $d \mathrm{~s}=-1.58,-1.86,-1.39$, and $-0.99 ; p \mathrm{~s}<.05]$. None of the pairwise comparisons in the both conditions reached significance $(p s>.10)$ when the target was present. For target-absent comparisons, the difference between the both 69 and the both 18 conditions was significant $[t(16)=-3.65, p<.05, d=-0.98]$, and the difference between the both 87 and the both 18 conditions was marginally significant $[t(16)=-1.94, p<.1$, $d=-0.64]$. Thus, there was some hint that there might be differences in performance across real-line stimuli of different contrasts, but after correction for multiple comparisons, the evidence was weak at best.

\section{Discussion}

In this experiment, search performances between the both conditions for all four gray levels of real contour were not significantly different from one another. In con-
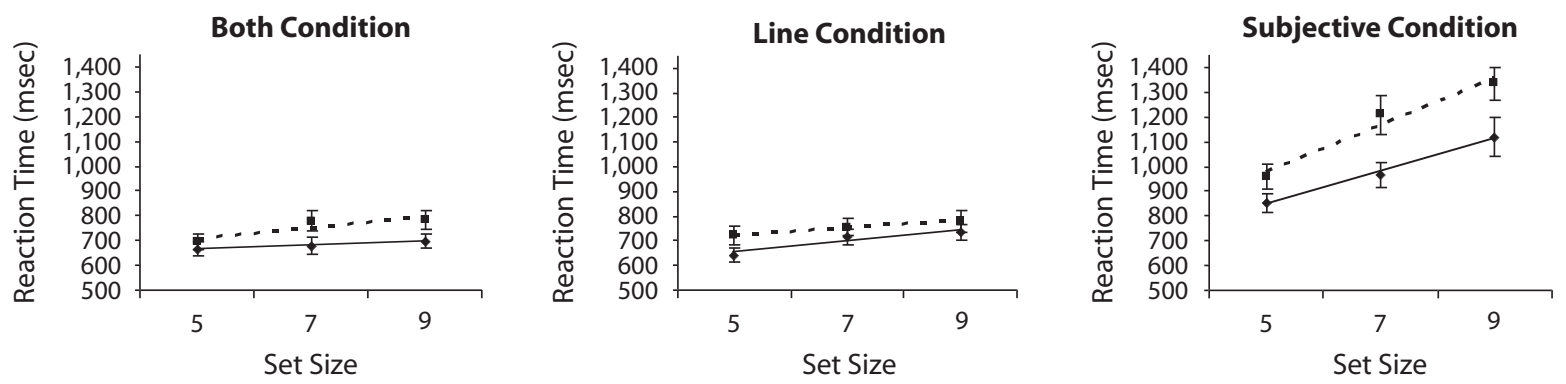

Figure 6. Reaction times in Experiment 4. Target-present trials are indicated by solid lines, and target-absent trials by dashed lines. 
trast, the performances in all four both conditions with different gray levels, even the faintest one, were significantly different from those in the subjective condition. The main conclusion from this experiment was that search performance with subjective contours was significantly worse than that with any of the real contours, even for the lowest contrast. If the subjective contours were simply acting as weak versions of real contours, they would have had to be substantially weaker than the $18 \%$ contrast contours.

\section{GENERAL DISCUSSION OF EXPERIMENTS 1-5}

Experiments 1-5 compared performance in searches for a specific shape among other distractor shapes. The shapes were induced by real contours (the line condition), Kanizsa-type subjective contours (the subjective condition), or a combination of both (the both condition). In Experiments 1 and 4, the participants searched for a horizontal rectangle among vertical rectangles. In Experiment 2, they searched for a square among triangles. Experiment 3 tested the effect of placeholders. In Experiment 5 , contours with different gray levels were used in the both conditions.

There is little evidence that Kanizsa-type subjective contours can define shapes that, in turn, will support efficient visual search. Slopes for the subjective condition were always inefficient with target-present slopes of greater than $20 \mathrm{msec} /$ item in all five experiments. The addition of relatively faint lines to the displays in the both condition immediately renders the search quite efficient, with slopes between 6 and $11 \mathrm{msec} /$ item. There is a faint glimmer of evidence for a role of subjective contours in the comparison of the line and the both conditions. In Experiments 1-4, target-present slopes in the line condition were always steeper than those in the corresponding both condition. Although this is a consistent effect, it does not always rise to statistical significance, and it may be due to the fact that the high-contrast pacmen in the both condition will enhance part of the contour of vertical and horizontal items, even if there is no role for the subjective contours induced by those pacmen. The safest conclusion would seem to be that the orientation features produced by the Kanizsa-type subjective contours were not able to guide the deployment of attention.

These results are consistent with those in Grabowecky and Treisman (1989) and are in agreement with those in Gurnsey et al. (1996) but are inconsistent with the results in Davis and Driver (1994). As was explained in the introduction, Davis and Driver's (1994) results may have been due to factors other than the perception of subjective contour. Our results were also different from those in Davis and Driver (1998). As was noted above, Davis and Driver's (1998) paradigm was quite indirect. They used three pacmen to induce a subjective rectangle and relied on the subjective figures acting as occluding surfaces. Our more direct approach has led to the conclusion that Kanizsa-type subjective contours cannot support efficient search.

\section{SUBJECTIVE CONTOURS INDUCED BY LINE ENDS}

Although Experiments 1-5 have shown that Kanizsatype subjective contours cannot guide attentional deployment, the perception of subjective contours induced by line ends may be different (Gurnsey et al., 1992). In Experiments 6 and 7, we used line end stimuli with the same methods as those employed in Experiments 1-5 so that we could directly compare the two types of stimuli.

\section{Experiment 6}

The task in Experiment 6 was to find one rectangle that had a different orientation from the rest. In this case, all the rectangles appeared on a background of black and white lines.

\section{Method}

Stimuli and Procedure. The stimuli are shown in Figure 7. The subjective contours were induced by interlaced black and white line segments on a gray background $\left(57 \mathrm{~cd} / \mathrm{m}^{2}\right)$. All of the targets and distractors were rectangles, whose ratio of length and width was $3: 1$. The distance between inducing lines was $0.28^{\circ}$. In the subjective condition, the stimuli were induced by line ends only. In the both condition, at the exact positions of the subjective contours, there were real contours of one of three gray levels: 16 , 39 , or $60 \mathrm{~cd} / \mathrm{m}^{2}$, producing contrasts of $56 \%, 19 \%$, or $3 \%$, respectively, for the both56, both19, and both 3 conditions. The task was to search for a target that was a rectangle slanting right, which was present on half of the trials. The set sizes were 5, 7, 9, or 13 . Twelve participants took part in this experiment. At the beginning of each trial, a red cross appeared at the center of the screen for $1 \mathrm{sec}$. The search array then appeared and was presented until response. Following 32 practice trials, there were 320 experimental trials in randomized order.
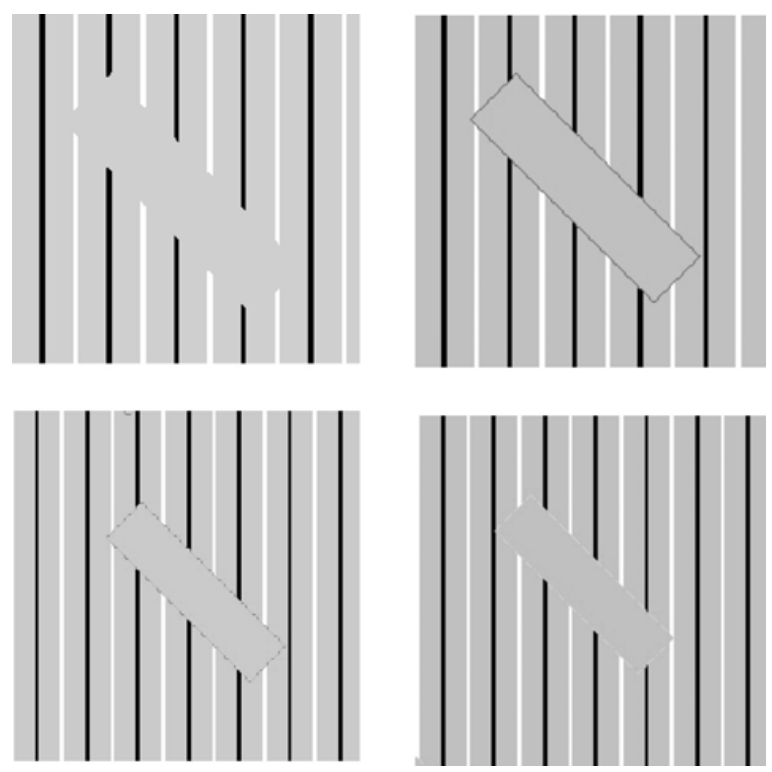

Figure 7. Part of the stimuli in Experiment 6. Top left: Subjective condition. Top right: Both56 condition. Bottom left: Both19 condition. Bottom right: Both3 condition. 


\section{Results}

In the both conditions, the slopes of the mean RT $\times$ set size functions (Figure 8) were $-1.08,-3.29$, and $-3.03 \mathrm{msec} /$ item for the both 56 , both 19 , and both 3 conditions, respectively, when the target was present and $7.40,12.77$, and $14.94 \mathrm{msec} /$ item when the target was absent. In the subjective condition, the target-present slope was $2.00 \mathrm{msec} / \mathrm{item}$, and the target-absent slope was $13.72 \mathrm{msec} /$ item.

A series of pairwise $t$ tests were conducted on the search slope data. All of the possible pairs of conditions were compared. None of these $t$ tests reached significance $(p \mathrm{~s}>.1)$. We carried out a two-way within-subjects ANOVA on the RT slopes, with the factors of stimulus type and target presence or absence. There was a main effect of target presence $\left[F(1,11)=5.31, p<.05, \eta_{\mathrm{p}}^{2}=\right.$ 0.33 ] but no main effect of stimulus type $(F<1)$ and no interaction $(F<1)$.

\section{Discussion}

The results showed that search in both the subjective and the both conditions was efficient and that there were no differences in performance between the four conditions. These results showed that the search in an array of figures produced by subjective contours induced by line ends differed markedly from those produced by Kanizsatype subjective contours. The fact that there was no difference between the both and the subjective conditions indicates that subjective contours induced by line ends can be used to guide attentional deployment.

\section{Experiment 7}

In Experiment 6, the ratio between length and width of the rectangles was $3: 1$, and the rectangles were tilted either $45^{\circ}$ left or $45^{\circ}$ right from vertical. These elements were different from those in Experiments 1 and 3. To test whether the differences across experiments were due to differences in the stimulus elements, we adjusted the stimuli in Experiment 7 to match those in Experiments 1 and 3. Experiment 7 was preceded by a pilot experiment that guided the choice of stimulus properties.

\section{Method}

Pilot study to explore perceptual strength of different types of contours. Various aspects of the inducing stimuli can affect the perceptual strength of subjective contours (Soriano, Spillmann, \& Bach, 1996). To make this experiment more comparable with Experiment 3, we matched the perceptual strength of the subjective contour stimuli in this experiment with those in Experiment 3. A pilot study was designed to determine the line width, line distance, and contrast of the darker lines and lighter lines of the background. In the pilot study, 17 participants were recruited to compare the strength of the contour of a Kanizsa-type subjective rectangle and a subjective rectangle induced by line ends, which were displayed side by side on the same display. On the left of the display, the Kanizsa-type subjective contour was kept constant and was similar to that used in Experiment 3. On the right of the display, there was a rectangle of the same size that was defined by subjective contours induced by line

\section{Both56 Condition}

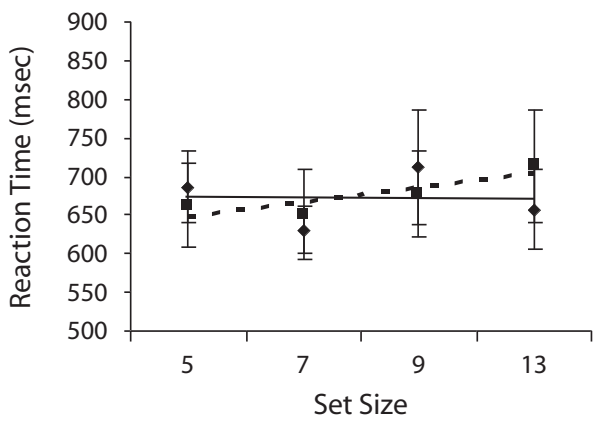

Both3 Condition

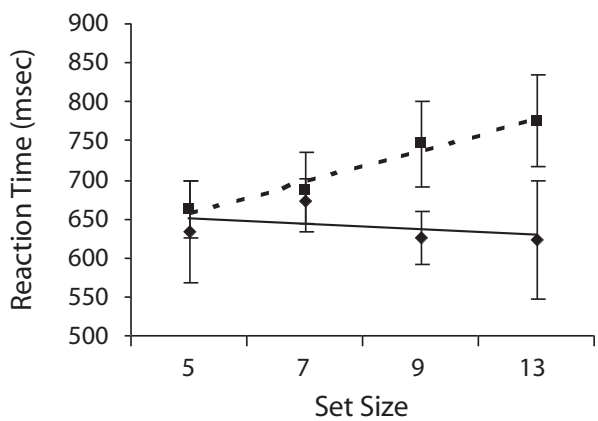

Both 19 Condition

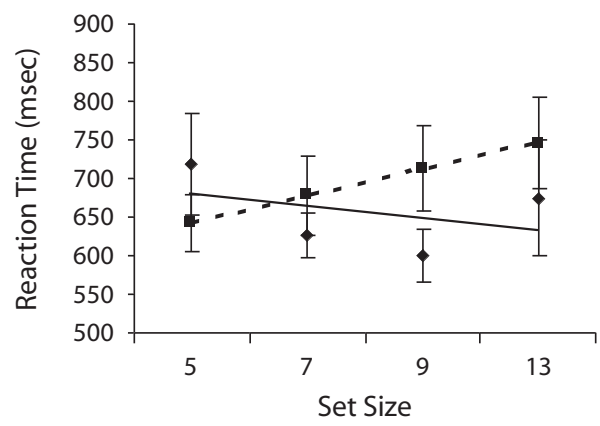

Subjective Condition

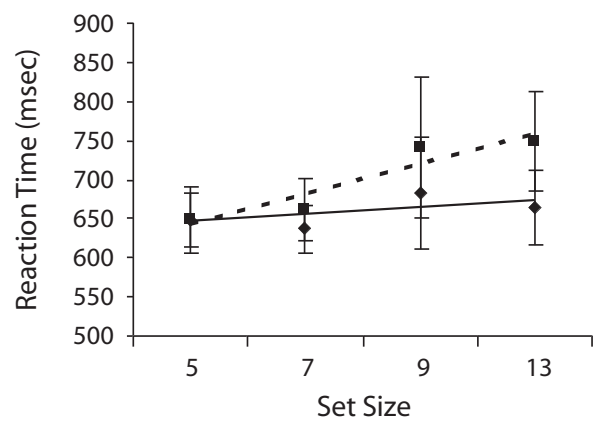

Figure 8. Reaction times in Experiment 6. Target-present trials are indicated by solid lines, and targetabsent trials by dashed lines. 
ends. The lines that induced the subjective contour varied in width $\left(2,3,4\right.$, or 5 pixels, extending over $0.03^{\circ}, 0.04^{\circ}, 0.06^{\circ}$, or $0.07^{\circ}$ of visual angle), in the distance between them $(10,15,20$, or 25 pixels, extending over $0.14^{\circ}, 0.21^{\circ}, 0.28^{\circ}$, or $0.35^{\circ}$ of visual angle), and in the contrast between darker and lighter lines $(100 \%, 80 \%$, or $60 \%$ ). Each participant was exposed once to every combination of these settings in each of three blocks. The participants were asked to report which rectangle had a stronger contour or, alternatively, to report that both had the same strength, by pressing one of three keys. The results showed that the participants were quite consistent in their responses across the three blocks $(52 \%$ of the reports were completely consistent; $36 \%$ were consistent across two blocks, and in these cases, the response in the third block was not the reverse to the other two). Because the responses in each of the three blocks were different, $4 \%$ of the reports were not included in the analysis. The response used in the analysis was the response that was given consistently in at least two blocks among the three blocks. These ratings from individual participants were then combined to pick one combination of contrast, line width, and line distance so that the line-ending contours matched the Kanizsa-type contours in strength. Of all the combinations, only two were rated as equal in strength to the Kanizsa-type contour by 8 of the 17 participants. (No combination was rated as equal by more than 8 participants.) Between these two combinations, the choice was made on the basis of the responses from the remaining 8 participants. One combination was chosen because it had a better balance between the number of participants that perceived the subjective contour to be stronger and the number that perceived the line-ending contour to be stronger. The best setting, which was used in Experiment 7, had a 3-pixel line width, a 20-pixel line distance, and the strongest contrast (100\% in contrast). For this best-matching condition, 8 of 17 participants agreed that the subjective contour induced by line ends had the same perceptual strength as that used in Experiment 3; 5 thought Kanizsa-type subjective contours were stronger; and 4 thought subjective contours induced by line ends were stronger.

The results of this pilot experiment determined the stimuli for Experiment 7, as described below.

Stimuli and Procedure. In this experiment, the task was to search for a horizontal rectangle among vertical rectangles. The ratio of the length and width of the rectangle was $1.5: 1$, which was similar to that used in Experiment 1. The stimuli are shown in Figure 9 . The line width was 3 pixels $\left(0.04^{\circ}\right)$; the line distance was 20 pixels $\left(0.28^{\circ}\right)$. There were three conditions in this experiment. In the subjective condition, the subjective contours were induced by line ends. In the both 81 and both 3 conditions, the rectangles were defined by both real contours and subjective contours. The contours in the both 81 condition $\left(6 \mathrm{~cd} / \mathrm{m}^{2}\right.$, producing a contrast of $\left.81 \%\right)$ were darker than the background, whereas the contours in the both 3 condition $\left(60 \mathrm{~cd} / \mathrm{m}^{2}\right.$, producing a contrast of $\left.3 \%\right)$ were lighter than the background. There were three blocks, each with a different stimulus type. The order of these three different blocks was balanced across different participants. In each block, there were 32 practice trials and 80 experimental trials. All of the other aspects of the stimuli

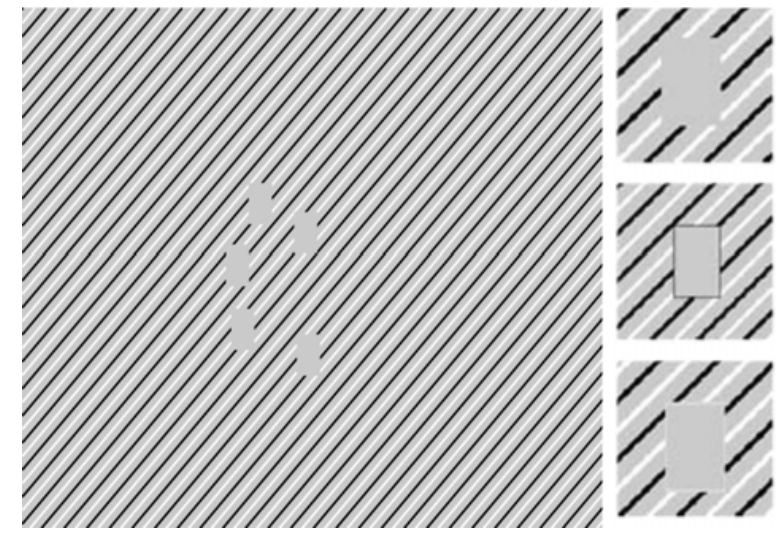

Figure 9. Stimuli in Experiment 7. Left: A search array. Right top: One of the rectangles in the subjective condition. Right middle: Rectangle in the both81 condition, with the real contour being darker than the background. Right bottom: Rectangle in the both 3 condition, with the real contour being lighter than the background.

were similar to those in Experiment 1. The positions for the corners of each rectangle were determined using the same method as the method for positioning the center of the pacmen in Experiment 1, which produces clumps of equal density across the different set sizes. The procedure was similar to that in Experiment 6.

\section{Results}

The results are shown in Figure 10. In the both81 condition, the mean slope for RT as a function of set size was $13.2 \mathrm{msec} / \mathrm{rectangle}$ when the target was present and $38.8 \mathrm{msec} / \mathrm{rectangle}$ when the target was absent. In the both 3 condition, the mean slope was $14.2 \mathrm{msec} /$ rectangle when the target was present and $34.4 \mathrm{msec} /$ rectangle when the target was absent. In the subjective condition, the slope was $13.0 \mathrm{msec} / \mathrm{rectangle}$ when the target was present and was $46.1 \mathrm{msec} / \mathrm{rectangle}$ when the target was absent.

A series of pairwise $t$ tests was conducted on the search slope data. In both the target-present and the target-absent conditions, none of the possible pairwise $t$ tests were significant $(p>.05)$. We carried out a two-way withinsubjects ANOVA on the RT slopes, with the factors of stimulus type and target presence. There was a main effect of target presence $\left[F(1,17)=20.93, p<.01, \eta_{\mathrm{p}}^{2}=\right.$
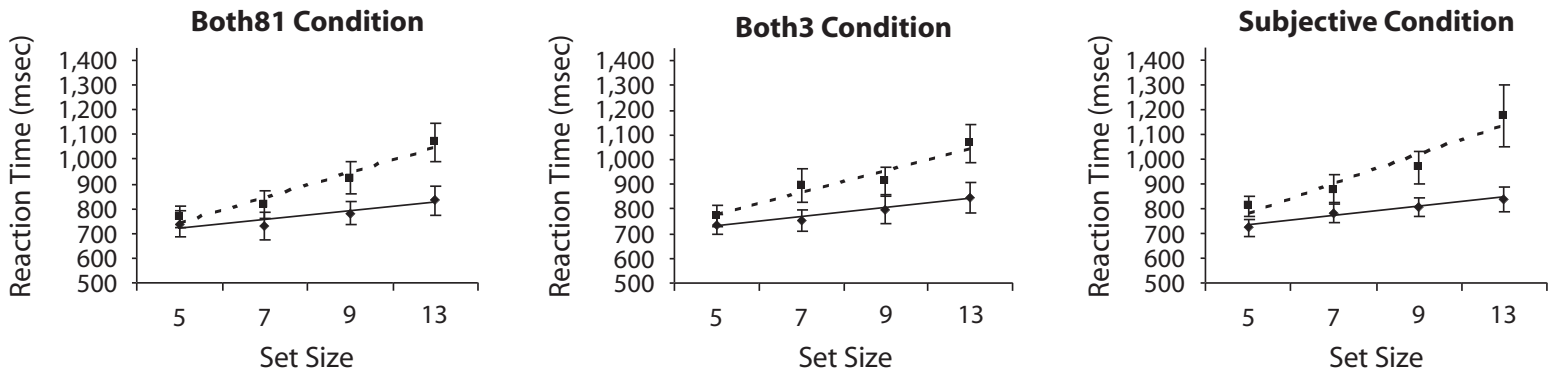

Figure 10. Reaction times in Experiment 7. Target-present trials are indicated by solid lines, and target-absent trials by dashed lines. 
$0.55]$ but no main effect of stimulus type $(F<1)$ and no interaction $[F(2,34)=1.2, p>.1]$.

\section{Discussion}

In this experiment, the search was fairly efficient in all of the stimulus type conditions. Moreover, the search performance was not significantly different between conditions.

The search performance with subjective contours induced by line ends apparently differs from that with Kanizsa-type subjective contours. The difference is unlikely to be due to the difference of perceptual strength between these two kinds of subjective contours. Although it is impossible to match the perceptual strength of these two kinds of subjective contour completely, we made extensive efforts to match the perceptual strength of the line-ending contours to that of the Kanizsa-type subjective contours in Experiment 3. The sizes of the targets and distractors were similar; the backgrounds were both gray; no placeholders was presented in either experiment; and the line width, line distance, and contrast of the background lines were determined by a pilot experiment to match the perceptual strength.

In Experiment 7, search performance was less efficient than in Experiment 6, indicating that the task in Experiment 7 was harder than that in Experiment 6 . However, for the purposes of the present article, the important point is that there was no difference in performance between search for orientation based on subjective and real contours.

\section{GENERAL DISCUSSION}

Using a visual search paradigm, this study took a new approach to the problem of whether perception of subjective contours can guide attention or not. Instead of only looking at the absolute RT slopes, we compared search performance with subjective contours against performance with comparable real contours, with all other factors equated across these conditions. Moreover, instead of asking whether the presence of a subjective contour attracted attention, we asked whether a difference in orientation or shape, defined by a subjective contour, could guide attention as effectively as the equivalent orientation or shape defined by a low-contrast luminance contour. Experiments 1-5 showed that visual search with Kanizsatype subjective contours was inefficient, whereas search for items defined by luminance contours alone or in combination with pacmen was much more efficient. As was noted earlier, this result is consistent with the conclusions of Grabowecky and Treisman (1989) and Gurnsey et al. (1996) but is inconsistent with those of Davis and Driver (1994, 1998). As has been argued by Gurnsey et al. (1996), the method used by Davis and Driver (1994) was quite indirect, and their efficient search among figures formed by Kanizsa-type subjective contours may have been an effect of factors other than subjective contours.

Using the same paradigm, we showed that visual search with subjective contours induced by line ends was efficient. RT slopes in the line, both, and subjective conditions did not differ. Consistent with previous studies (Gurnsey et al., 1992), this result shows that subjective contour induced by line ends can effectively guide attention. Our results add to the previous work by showing a difference between the two types of subjective contours, using the same methods and with the two types of contour equated, as best possible, for perceptual salience.

In this study, visual searches with Kanizsa-type subjective contours were inefficient, suggesting that attention must be narrowly focused to find the target. Some studies have shown that grouping is crucial for Kanizsa-type subjective contour perception (Fahle \& Koch, 1995), and other studies have shown that grouping may require attention (Driver, Davis, Russell, Turatto, \& Freeman, 2001). It is possible that the procedure of grouping pacmen together requires attention.

The finding that Kanizsa-type subjective contours and subjective contours induced by line ends perform differently in guiding attentional deployment is consistent with neurophysiological studies suggesting that these two types of subjective contours may be detected by different neural structures. Von der Heydt, Peterhans, and Baumgartner (1984) demonstrated that some V2 neurons respond to both types of subjective contours. They found no evidence of responses to either type of subjective contour in V1, but a later study by Grosof, Shapley, and Hawken (1993) included V1 cells that responded to line end subjective contours. If line end contours can be detected in V1 but Kanizsa-type subjective contours cannot be detected until V2, these two kinds of subjective contours are detected by different mechanisms at different stages of visual processing, and it is thus less surprising that these subjective contours perform differently when guiding attention.

\section{AUTHOR NOTE}

We thank Keith Rayner and Neil E. Berthier for useful suggestions. We also thank Nick Cammuso for help in conducting the experiments. Thanks to Rick Gurnsey for his tolerance and advice and an anonymous reviewer for other helpful suggestions. Part of this study was presented at OPAM 2004. Requests for reprints should be addressed to K. R. Cave, Department of Psychology, University of Massachusetts, Amherst, MA 01003 (e-mail: kcave@psych.umass.edu) or to X. Li, Department of Psychology, Vanderbilt University, 301 Wilson Hall, 111 21st Avenue South, Nashville, TN 37203 (e-mail: xingshan.li@vanderbilt.edu).

\section{REFERENCES}

Carrasco, M., \& Frieder, K. S. (1997). Cortical magnification neutralizes the eccentricity effect in visual search. Vision Research, 37, 63-82.

Cavanagh, P., Arguin, M., \& Treisman, A. (1990). Effect of surface medium on visual search for orientation and size features. Journal of Experimental Psychology: Human Perception \& Performance, 16, 479-491.

Cave, K. R. (1999). The FeatureGate model of visual selection. Psychological Research, 62, 182-194.

DAVIS., G., \& DrIVER., J. (1994). Parallel detection of Kanizsa subjective figures in the human visual system. Nature, 371, 791-793.

DAvis, G., \& Driver, J. (1998). Kanizsa subjective figures can act as occluding surfaces at parallel stages of visual search. Journal of Experimental Psychology: Human Perception \& Performance, 24, 169-184.

Driver, J., Davis, G., Russell, C., Turatto, M., \& Freeman, E. (2001). Segmentation, attention and phenomenal visual objects. Cognition, 80, 61-95.

Egeth, H. E., Virzi, R. A., \& Garbart, H. (1984). Searching for 
conjunctively defined targets. Journal of Experimental Psychology: Human Perception \& Performance, 10, 32-39.

Fahle, M., \& Koch, C. (1995). Spatial displacement, but not temporal asynchrony, destroys temporal binding. Vision Research, 35, 491-494.

Grabowecky, M., \& Treisman, A. (1989). Attention and fixation in subjective contour perception. Investigative Ophthalmology \& Visual Science, 30(Suppl.), 457.

Green, B. F., \& ANDERson, L. K. (1956). Color coding in a visual search task. Journal of Experimental Psychology, 51, 19-24.

Grosof, D. H., Shapley, R. M., \& Hawken, M. J. (1993). Macaque V1 neurons can signal "illusory" contours. Nature, 365, 550-552.

Gurnsey, R., Humphrey, G. K., \& Kapitan, P. (1992). Parallel discrimination of subjective contours defined by offset gratings. Perception \& Psychophysics, 52, 263-276.

Gurnsey, R., Poirier, F. J. A. M., \& Gascon, E. (1996). There is no evidence that Kanizsa-type subjective contours can be detected in parallel. Perception, 25, 861-874.

Herrmann, C. S., \& Mecklinger, A. (2000). Magnetoencephalographic responses to illusory figures: Early evoked gamma is affected by processing of stimulus features. International Journal of Psychophysiology, 38, 265-281.

LESHER, G. W. (1995). Illusory contours: Toward a neurally based perceptual theory. Psychonomic Bulletin \& Review, 2, 279-321.

Rock, I., \& Anson, R. (1979). Illusory contours as the solution to a problem. Perception, 8, 665-681.

Senkowski, D., Röttger, S., Grimm, S., Foxe, J. J., \& Herrmann, C. S. (2005). Kanizsa subjective figures capture visual spatial atten- tion: Evidence from electrophysiological and behavioral data. Neuropsychologia, 43, 872-886.

ShaPley, R. [M.], \& GoRDON, J. (1985). Nonlinearity in the perception of form. Perception \& Psychophysics, 37, 84-88.

Soriano, M., SPILlmann, L., \& BaCH, M. (1996). The abutting grating illusion. Vision Research, 36, 109-116.

Takahashi, S., Ohya, K., Arakawa, K., \& Tanabe, Y. (2007). Luminance differences between the figures and background are not necessary for parallel search of a Kanizsa illusory square. Japanese Psychological Research, 49, 45-56.

Townsend, J. T. (1990). Serial vs. parallel processing: Sometimes they look like Tweedledum and Tweedledee but they can (and should) be distinguished. Psychological Science, 1, 46-54.

VON DER HEYDT, R. (1987). Approaches to visual cortical function. Reviews of Physiology, Biochemistry, \& Pharmacology, 108, 69-150.

von der Heydt, R., Peterhans, E., \& Baumgartner, G. (1984). Illusory contours and cortical neuron responses. Science, 224, 1260-1262.

Wolfe, J. M. (1998). Visual search. In H. Pashler (Ed.) Attention (pp. 13-73). Hove, U.K.: Psychology Press.

WolfE, J. M. (2003). Moving towards solutions to some enduring controversies in visual search. Trends in Cognitive Sciences, 7, 70-76.

Wolfe, J. M., Horowitz, T. S., \& Michod, K. O. (2007). Is visual attention required for robust picture memory? Vision Research, 47, 955-964.

(Manuscript received March 1, 2006; revision accepted for publication October 18, 2007.) 